\section{A CROSS COUNTRY ANALYSIS OF DROWNING IN SRI LANKA: 2001 TO 2006 AND 2009}

${ }^{1}$ Bernadette Matthews, ${ }^{1}$ Rhiannon Birch, ${ }^{1}$ Mevan Jayawardena, ${ }^{2}$ Dushani Mathew ${ }^{2}$ Asanka Nanayakkara, ${ }^{2}$ Sanath Wiyayaratne, ${ }^{3}$ Samath D Dharmaratne. 'Life Saving Victoria; ${ }^{2}$ Life Saving Association of Sri Lanka; ${ }^{3}$ Department of Community Medicine, Faculty of Medicine, University of Peradeniya, Sri Lanka

\subsection{6/injuryprev-2016-042156.824}

Introduction Drowning is a major cause of death and injury in Sri Lanka. Published data on the number and causes of drowning incidents of provinces are scarce. Therefore, we conducted this analysis to describe the burden of drowning in Sri Lanka from 2001 to 2006 and 2009 by province.

Methods Data from the first drowning report, 'Drowning prevention report Sri Lanka', published in December, 2014 by the Life Saving Association of Sri Lanka and Life Saving Victoria was used in this analysis. This report includes unintentional drowning deaths reported in Sri Lanka during the study period.

Results Sri Lanka consists of nine provinces, with the North Central being the largest (16\% of total area) and the Western the smallest $(5.6 \%$ of total area). The highest proportion of the population $(28.6 \%)$ live in the Western province and the smallest in the Northern province (5.6\%). Each year, an estimated 236 people die in the Western province from drowning while 44 die in the Uwa and the Northern provinces. The death rate is highest in the North Western province $(6.3$ per 100,000$)$ and lowest in the Central province $(3.4$ per 100,000). North Western (6.3), North Central (5.4) and Southern (4.2) provinces had a higher drowning death rate than the national average $(4.4$ per 100,000$)$. Even in some of the provinces adjoining the ocean (Eastern and Northern), the commonest location of drowning was reportedly lakes and wells.

Conclusion Significant cross country differences identified in this analysis should be used by policy makers to prevent deaths from drowning in Sri Lanka.

\section{THE BURDEN OF DROWNING IN SRI LANKA: 2001 TO 2006 AND 2009}

${ }^{1}$ Bernadette Matthews, ${ }^{1}$ Rhiannon Birch, ${ }^{1}$ Mevan Jayawardena, ${ }^{2}$ Dushani Mathew, ${ }^{2}$ Asanka Nanayakkara, ${ }^{2}$ Sanath Wiyayaratne, ${ }^{3}$ Samath D Dharmaratne. 'Life Saving Victoria; ${ }^{2}$ Life Saving Association of Sri Lanka; ${ }^{3}$ Department of Community Medicine, Faculty of Medicine, University of Peradeniya, Sri Lanka

\subsection{6/injuryprev-2016-042156.825}

Introduction Drowning is a major cause of death and injury in Sri Lanka. Published data on the number and causes of drowning incidents are scarce. Therefore, we undertook this analysis to describe the burden of drowning in Sri Lanka using available data from 2001 to 2006 and 2009.

Methods Data from the first drowning report, 'Drowning prevention report Sri Lanka', published in December, 2014 by the Life Saving Association of Sri Lanka and Life Saving Victoria was used in this analysis. This report includes unintentional drowning deaths reported in Sri Lanka during the study period.

Results During study period, on average, 855 people died from drowning each year, producing a drowning rate of 4.4 deaths per 100,000 persons. Males were four times more likely to drown than females. Adults aged 25 to 44 years had the highest number of drowning deaths, followed by the 45 to 64 year group, but the age specific death rate was highest in those aged 65 years and over (8.25 deaths per 100,000). Lakes, wells and open cisterns, and the ocean were the commonest reported drowning locations in Sri Lanka.

Conclusions Globally, the drowning rate of Sri Lanka is ranked $12^{\text {th }}$ in a comparison of 61 countries, highlighting that this is an important public health problem. Middle aged males are mainly affected, therefore intervention strategies are needed to target this group. Significantly, the highest risk location is lakes, although Sri Lanka is an island. This analysis, a first of its kind, should be used by policy makers to control and prevent deaths from drowning in Sri Lanka.

\section{ANALYSING A DROWNING CASE IN COX'S BAZAR BEACH IN BANGLADESH: IMPLICATION FOR FUTURE INTERVENTION}

${ }^{1}$ Salim Mahmud Chowdhury, ${ }^{1}$ Jahangir Hossain, ${ }^{2}$ Steve Wills, ${ }^{1}$ Fazlur Rahman. ${ }^{1}$ Centre for Injury Prevention and Research, Bangladesh (CIPRB), ${ }^{2}$ Royal National Lifeboat Institution (RNLI)

\subsection{6/injuryprev-2016-042156.826}

Background Beach tourism has become very popular in many countries including Bangladesh. However, number people dying in these beaches due to lack of safety measures. In this study, we aimed at identifying possible solution in preventing beach drowning.

Methods An in-depth analysis (case study) of a drowning case of Cox's Bazar beach, Bangladesh was analysed for this study.

Findings Mishu, a university student was on holiday in Cox's Bazar with his family. On 06 September 2014, he went with his family members to the nearest beach to the hotel where they were staying. Mishu, along with his four cousins, agreed to go into the sea to the chest depth. None of them knew how to swim except Minhaz one of the cousins. They went under the rolling and turbulent water when a large wave came in and broke over their heads. Minhaz tried to help Mishu by pulling him back to the shore. But, he couldn't as Mishu was quite heavy. Mishu was under the water for about one minute. A patrolling SeaSafe lifeguard and another SeaSafe lifeguard in the watching tower observed the incidence. Both lifeguards rescued the victims from the water and kept the victims in the recovery position at the shore. Mishu started to vomit blood while he was in recovery position. Unfortunately, there was no ambulance available to transfer him to a hospital. So, the lifeguards hired an auto. After arriving at the hospital, he was kept in the recovery position and oxygen and an intravenous saline drip 10 minutes after his arrival. His family members were requested to fill in various forms before they started treatment. After few minutes, he became conscious and talked to his relatives. There was no doctor in the emergency department at that time. As the hospital lacked any expertise to help drowning victims immersed in saline water, Mishu's family was requested to transfer him to the nearest (143 km from Cox's Bazar) tertiary level hospital for further treatment. Mishu's condition worsened and he died within two hours of his arrival in the hospital.

Conclusion Analysis of this case could be a think point for enforcing beach patrolling by lifeguards (with lifesaving skills) and establishment of emergency medical care with ambulance services. 